\title{
Fluorine in extremely hot post-AGB stars: Evidence for nucleosynthesis ${ }^{\star}$
}

\author{
K. Werner ${ }^{1}$, T. Rauch ${ }^{1,2}$, and J. W. Kruk ${ }^{3}$ \\ ${ }^{1}$ Institut für Astronomie und Astrophysik, Universität Tübingen, Sand 1, 72076 Tübingen, Germany \\ e-mail: werner@astro.uni-tuebingen.de \\ ${ }^{2}$ Dr.-Remeis-Sternwarte, Universität Erlangen-Nürnberg, Sternwartstraße 7, 96049 Bamberg, Germany \\ 3 Department of Physics and Astronomy, Johns Hopkins University, Baltimore, MD 21218, USA
}

Received 26 October 2004 / Accepted 30 November 2004

\begin{abstract}
We have discovered lines of highly ionized fluorine (F V and F VI) in the far-UV spectra of extremely hot $\left(T_{\text {eff }}=85000-150000 \mathrm{~K}\right)$ post-AGB stars. Our sample comprises H-rich central stars of planetary nebulae as well as H-deficient PG1159 stars. We performed non-LTE calculations and find strong F overabundances (up to $10^{-4}$ by mass, i.e., 250 times solar) in a number of PG1159 stars, while F is essentially solar in the H-rich stars. Since PG1159 stars are believed to exhibit intershell matter of the preceding AGB phase on their surface, their chemical analyses allow for a direct insight into nucleosynthesis processes during the AGB phase. The high F abundances in PG1159 stars confirm the conclusion from abundance determinations in giants, that $\mathrm{F}$ is synthesized in AGB stars and that the $\mathrm{F}$ enrichment in the intershell must be very high.
\end{abstract}

Key words. stars: abundances - stars: evolution - stars: AGB and post-AGB - stars: white dwarfs

\section{Introduction}

For a long time, the solar system was the only location of the Galaxy with known fluorine $\left({ }^{19} \mathrm{~F}\right)$ abundance (see e.g. Meynet $\&$ Arnould 2000). The production site of $F$ has always been a puzzle, although its abundance is very small ( $\log \mathrm{F}=-6.4$ by mass; Grevesse \& Sauval 1998). This situation changed since the detection of $\mathrm{F}$ overabundances (up to 30 times solar) in red giants (Jorissen et al. 1992) with the discovery of an infrared HF molecule line. This proves that AGB stars are in fact F producers, but the question is still open as to what extent AGB stars contribute to the solar-system and Galactic F content. A recent analysis of HF lines in giants in $\omega$ Cen, though, suggests that AGB stars are not the main F contributor (Cunha et al. 2003).

Other potential $\mathrm{F}$ producers are Wolf-Rayet stars which increase the Galactic F content by wind mass-loss (Meynet \& Arnould 2000), and in type II supernovae ${ }^{19} \mathrm{~F}$ is created by spallation of ${ }^{20} \mathrm{Ne}$ by neutrinos (Woosley \& Weaver 1995). Renda et al. (2004) address the relative importance of these three primary astrophysical factories of fluorine production in the Galaxy.

The general problem of fluorine production is that ${ }^{19} \mathrm{~F}$, the only stable $\mathrm{F}$ isotope, is rather fragile and readily destroyed in hot stellar interiors by hydrogen via ${ }^{19} \mathrm{~F}(\mathrm{p}, \alpha){ }^{16} \mathrm{O}$ and helium

* Based on observations made with the NASA-CNES-CSA Far Ultraviolet Spectroscopic Explorer. FUSE is operated for NASA by the Johns Hopkins University under NASA contract NAS5-32985. via ${ }^{19} \mathrm{~F}(\alpha, \mathrm{p}){ }^{22} \mathrm{Ne}$. The nucleosynthesis path for $\mathrm{F}$ production in He-burning environments of AGB and Wolf-Rayet stars is:

${ }^{14} \mathrm{~N}(\alpha, \gamma){ }^{18} \mathrm{~F}\left(\beta^{+}\right){ }^{18} \mathrm{O}(\mathrm{p}, \alpha){ }^{15} \mathrm{~N}(\alpha, \gamma){ }^{19} \mathrm{~F}$.

Protons are provided by the ${ }^{14} \mathrm{~N}(\mathrm{n}, \mathrm{p}){ }^{14} \mathrm{C}$ reaction with neutrons liberated from ${ }^{13} \mathrm{C}(\alpha, \mathrm{n}){ }^{16} \mathrm{O}$. The ${ }^{14} \mathrm{~N}$ and ${ }^{13} \mathrm{C}$ nuclei can result from ashes of hydrogen burning by $\mathrm{CNO}$ cycling. By this, ${ }^{19} \mathrm{~F}$ is enriched in the convective He-rich intershell of AGB stars and then dredged-up to the surface (3rd dredge up). However, if there is no other source of ${ }^{14} \mathrm{~N}$ and ${ }^{13} \mathrm{C}$ nuclei than the ashes of hydrogen burning, then ${ }^{19} \mathrm{~F}$ will be produced in a negligible amount only. To yield the observed overabundances, primary ${ }^{13} \mathrm{C}$ and ${ }^{14} \mathrm{~N}$ nuclei are an absolute necessity. They probably result from proton mixing ("partial mixing") into the He-rich intershell, the same mixing necessary to activate the s-process in those stars (Mowlavi et al. 1998).

Current stellar models cannot match the highest F abundances observed in AGB stars (Lugaro et al. 2004). The problems in modeling arise from the mixing and burning processes during thermal pulses, and still uncertain nuclear reaction rates, particularly for a competing process that prevents the F synthesis: the ${ }^{15} \mathrm{~N}(\mathrm{p}, \alpha){ }^{12} \mathrm{C}$ reaction removes ${ }^{15} \mathrm{~N}$ and protons from the $\mathrm{F}$ production chain. In order to explain the highest observed $\mathrm{F}$ enrichments, one would require very large $\mathrm{F}$ overabundances in the intershell $\left(10^{-3}-10^{-4}\right.$, i.e. $250-2500$ times solar). The models do produce a fluorine overabundance, but not high enough.

In this paper we announce the detection of $\mathrm{F}$ in extremely hot post-AGB stars, which gives new insight into the problem 
Table 1. Parameters of the H-deficient program stars. Abundances are given in mass fractions. The solar $\mathrm{F}$ abundance is log $\mathrm{F}=-6.4$. We note that in some objects trace nitrogen (abundance of order 0.01) was found (see last column). Ne abundances from Werner et al. (2004a).

\begin{tabular}{|c|c|c|c|c|c|c|c|c|c|c|c|}
\hline Object & $\begin{array}{r}T_{\text {eff }} \\
{[\mathrm{kK}]}\end{array}$ & $\begin{array}{l}\log g \\
(\operatorname{cgs})\end{array}$ & $\mathrm{H}$ & $\mathrm{He}$ & $\mathrm{C}$ & $\mathrm{O}$ & $\mathrm{Ne}$ & $\log F$ & $\begin{array}{l}\text { Mass } \\
{\left[\mathrm{M}_{\odot}\right]}\end{array}$ & Ref. & Remark \\
\hline H1504+65 & 200 & 8.0 & & $<.01$ & .48 & .48 & .02 & $<-4.5$ & 0.84 & A & $\mathrm{Mg}=0.02$ \\
\hline RXJ2117.1+3412 & 170 & 6.0 & & .38 & .54 & .06 & .02 & $<-5.0$ & 0.70 & B & \\
\hline PG1520+525 & 150 & 7.5 & & .43 & .38 & .17 & .02 & -4.0 & 0.65 & $\mathrm{~J}$ & \\
\hline PG1144+005 & 150 & 6.5 & & .38 & .58 & .02 & .02 & -5.0 & 0.57 & $\mathrm{C}$ & Trace nitrogen \\
\hline NGC 246 & 150 & 5.7 & & .62 & .30 & .06 & .02 & -4.0 & 0.76 & $\mathrm{H}$ & \\
\hline PG1159-035 & 140 & 7.0 & & .33 & .48 & .17 & .02 & -5.5 & 0.54 & $\mathrm{I}, \mathrm{J}$ & \\
\hline $\mathrm{K} 1-16$ & 140 & 6.4 & & .33 & .48 & .17 & .02 & -6.5 & 0.59 & $\mathrm{G}$ & \\
\hline HS2324+397 & 130 & 6.2 & .21 & .41 & .37 & .01 & & $<-6.0$ & 0.59 & $\mathrm{E}$ & Poor $\mathrm{S} / \mathrm{N}$, strong i.s. $\mathrm{H}_{2}$ contamination \\
\hline Longmore 4 & 120 & 5.5 & & .45 & .42 & .11 & .02 & $<-6.5$ & 0.65 & $\mathrm{D}$ & \\
\hline PG1424+535 & 110 & 7.0 & & .49 & .43 & .06 & .02 & -4.0 & 0.50 & $\mathrm{~J}$ & \\
\hline NGC 7094 & 110 & 5.7 & .35 & .41 & .21 & .01 & .02 & -5.0 & 0.59 & $\mathrm{E}$ & $1139.5 \AA$ a wind profile \\
\hline Abell 78 & 110 & 5.5 & & .35 & .50 & .15 & & -5.0 & 0.77 & $\mathrm{~F}$ & $1139.5 \AA$ Aind profile; trace nitrogen \\
\hline PG1707+427 & 85 & 7.5 & & .43 & .38 & .17 & .02 & -4.0 & 0.54 & $\mathrm{~J}$ & poor $\mathrm{S} / \mathrm{N}$; trace nitrogen \\
\hline
\end{tabular}

References: A: Werner et al. (2004b), B: Werner et al. (1996), C: Werner \& Heber (1991), D: Werner et al. (1992), E: Dreizler et al. (1997), F: Koesterke \& Werner (1998), G: Kruk \& Werner (1998), H: Rauch \& Werner (1997), I: Werner et al. (1991), J: Dreizler \& Heber (1998).

and strongly confirms the role of AGB stars as Galactic F producers. We derive very high $\mathrm{F}$ overabundances in the hydrogendeficient stars of our sample, the so-called PG1159 stars. This is of particular interest, because these objects are thought to exhibit intershell abundances on their surface, probably as a consequence of a late He-shell flash (Werner 2001). An analysis of a representative subset of our full sample, for which we have spectra with particularly good $\mathrm{S} / \mathrm{N}$, will be presented here.

We are currently analysing a large sample of very hot postAGB stars in order to derive atmospheric parameters and to gain insight into the evolutionary history of these stars. In particular we are interested in the PG1159 stars because many aspects of their evolution are still unclear. UV spectroscopy with the Far Ultraviolet Spectroscopic Explorer (FUSE) is most suitable for this work because all metals in the hot photospheres are highly ionised. During the course of our analyses we realized that, although the spectra are poor in the number of line features (from highly ionized species, essentially $\mathrm{He}, \mathrm{C}, \mathrm{O}, \mathrm{Ne}$ ), a number of lines still cannot be identified. One of these features, located at $1139.5 \AA$, is detectable in most PG1159 stars, over a quite large temperature range $\left(T_{\text {eff }}=110000-150000 \mathrm{~K}\right)$. It is sometimes very prominent with central depressions of $50 \%$, and it is also seen in many $\mathrm{H}$-rich central stars with temperatures ranging between $70000-130000 \mathrm{~K}$. We identify this line as due to F VI, which is confirmed by the additional identification of a F V multiplet in the "coolest" PG1159 stars and H-rich central stars of our sample.

\section{Fluorine abundance analysis}

FUSE observations and data reduction for most of our program stars were described in a previous work (Werner et al. 2004a). Further observations utilized here will be described in the comprehensive work mentioned above.

We have designed a fluorine model atom for NLTE line formation calculations. These are performed using and
Table 2. Parameters of the H-rich stars. Number abundance ratios of $\mathrm{H}, \mathrm{He}, \mathrm{C}$, and $\mathrm{O}$ are given. The $\mathrm{F}$ abundance is in mass fraction; the solar abundance is $\log \mathrm{F}=-6.4$. Photospheric and stellar parameters are from Traulsen et al. (2005), except for NGC 1535 (Méndez et al. 1992).

\begin{tabular}{lrrrrrr}
\hline \hline Object & $\begin{array}{r}T_{\text {eff }} \\
{[\mathrm{kK}]}\end{array}$ & $\begin{array}{l}\log g \\
(\mathrm{cgs})\end{array}$ & $\mathrm{He} / \mathrm{H}$ & $\mathrm{C} / \mathrm{O}$ & $\log \mathrm{F}$ & $\begin{array}{r}\text { Mass } \\
{\left[M_{\odot}\right]}\end{array}$ \\
\hline NGC 1535 & 70 & 4.6 & 0.12 & & -6.5 & 0.65 \\
NGC 1360 & 97 & 5.3 & 0.25 & 1.0 & -7.0 & 0.65 \\
LSS 1362 & 114 & 5.7 & 0.10 & 1.0 & -7.0 & 0.60 \\
NGC 7293 & 120 & 6.3 & 0.03 & .86 & -6.5 & 0.56 \\
NGC 6853 & 126 & 6.5 & 0.10 & 2.0 & $<-6.0$ & 0.58 \\
\hline
\end{tabular}

keeping fixed the physical structure (temperature, densities) of line blanketed NLTE model atmospheres which are described in detail in Werner et al. (2004a). In short, they are plane-parallel and in hydrostatic and radiative equilibrium. For the PG1159 stars, the model parameters and references for the previous analyses are given in Table 1 . The models are composed of $\mathrm{H}, \mathrm{He}, \mathrm{C}, \mathrm{O}$, and $\mathrm{Ne}$. For the $\mathrm{H}$-rich central stars we calculated models with a solar composition, which is close but not identical to the literature values given in Table 2 (the model for NGC 1360 accounts for the oversolar He abundance). Due to convergence problems, the models for NGC 1360 and NGC 1535 do not include O and Ne. Both restrictions are not expected to have a strong influence on the calculated fluorine line profiles.

For each star we performed $\mathrm{F}$ calculations with different abundances in order to estimate the observed abundance and the error of the analysis. For two objects our static models are not adequate. NGC 7094 and Abell 78 show distinct extended blue wings in the FVI profile (Fig. 1), which clearly are the signature of mass-loss. Both stars also show strong P Cyg profiles in the C III and OVI resonance lines. Modeling of 


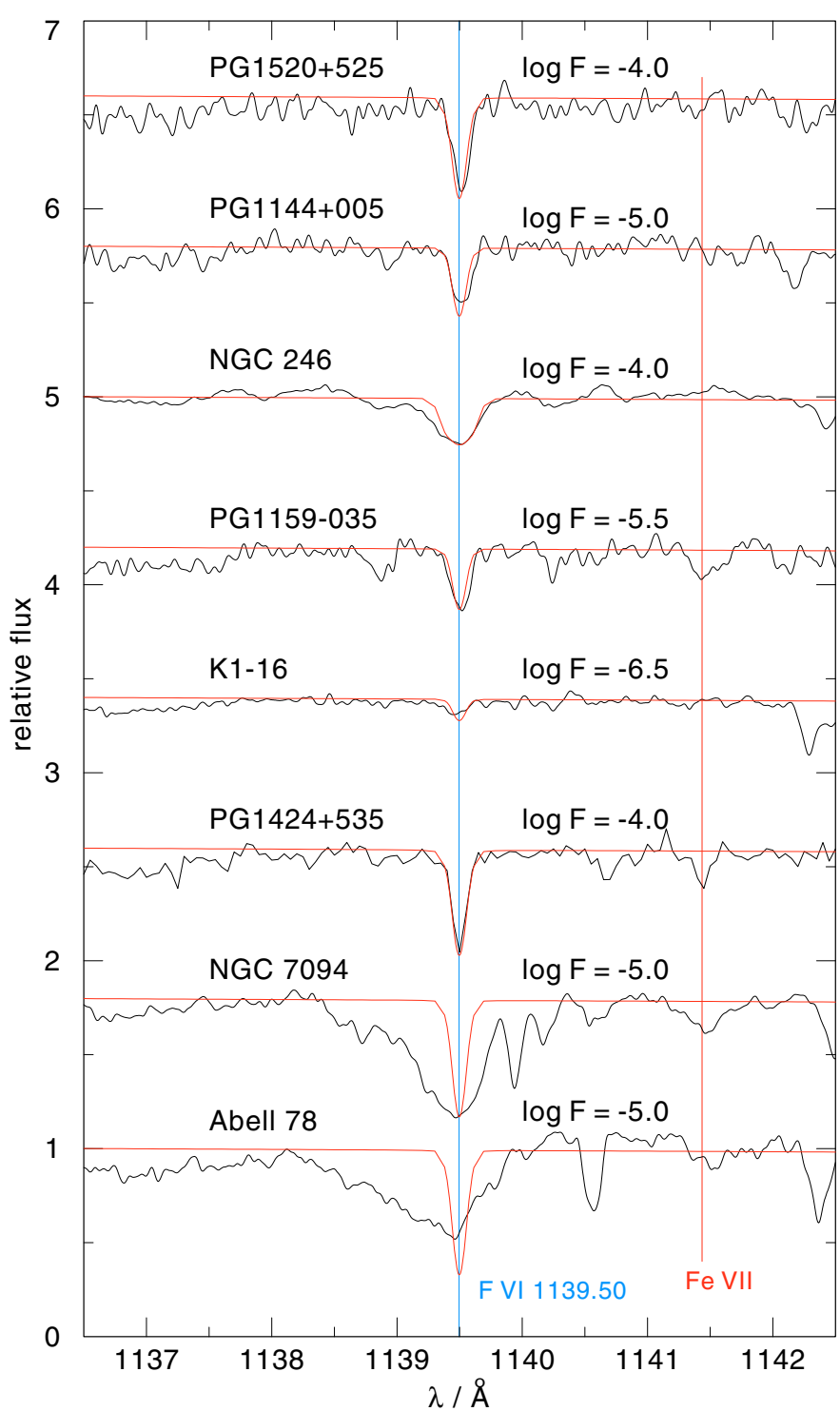

Fig. 1. Identification of the F VI $1139.5 \AA$ line in PG1159 stars. The $\mathrm{F}$ abundances of the overplotted models are given (the solar abundance is $\log \mathrm{F}=-6.4$ ). The observed line profiles of NGC 7094 and Abell 78 are significantly broader than the computed ones due to wind effects, which are neglected in our models. Rotational broadening $\left(45 \mathrm{~km} \mathrm{~s}^{-1}\right)$ is applied to the NGC 246 profile. The position of a Fe VII line is also marked. It is very weak or not detectable in the PG1159 stars because of their iron-deficiency (Miksa et al. 2002). Other lines are of interstellar origin.

expanding atmospheres is beyond the scope of this paper and we assign a larger error to the $\mathrm{F}$ abundance determination in these two cases.

The model atom considers ionization stages F IV-F VIII, represented by $2,8,6,2,1$ NLTE levels, respectively, plus a number of LTE levels (Fig. 3). In the ions F V-F VII we include 9, 4, 1 line transitions. Atomic data were taken from the NIST and Opacity Project (OP, Seaton et al. 1994) databases. Special attention was given to the lines which we compare to the observation. In order to compute the three profiles of the F V multiplet $(\lambda \lambda 1082.31 \AA, 1087.82 \AA, 1088.39 \AA)$, we performed fine structure splitting of energy levels and distributed level

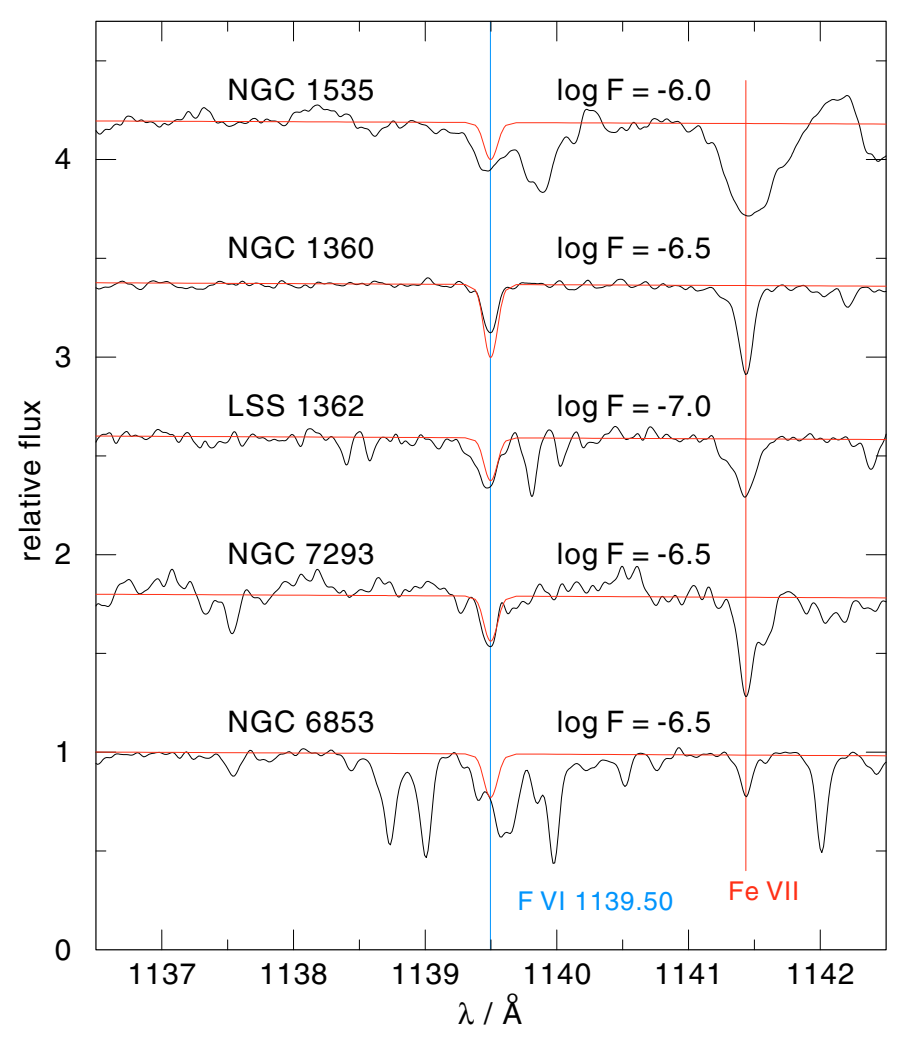

Fig. 2. Like Fig. 1, but here for the hydrogen-rich central stars. The finally adopted $\mathrm{F}$ abundances are listed in Table 2. The Fe VII line is strongly detected and probably consistent with solar $\mathrm{Fe}$ abundance (Hoffmann et al. 2005). It is not included in the models being plotted.

population densities according to statistical weights. For all lines we assumed quadratic Stark broadening for the profile calculation. Oscillator strengths are taken from OP.

It is remarkable that these F V and F VI lines are detectable in the observations, even at very low F abundances. This is because they are the only UV lines that arise from relatively low excited levels (excitation energy about $200000 \mathrm{~cm}^{-1}$ ). All other strong lines from these ions, arising from such low lying levels, are located in the inaccessible EUV spectral region.

In seven out of twelve PG1159 stars we identified the FVI $1139.5 \AA$ line. They are plotted in Fig. 1 together with synthetic profiles from those grid models with $\mathrm{F}$ abundances that most closely match the observations. In the two hottest PG1159 stars (H1504+65 and RX J2117.1+3412) F VI $1139.5 \AA$ is not detected because the ionization shifts away from F VI at these high temperatures, hence, only large upper limits for the abundance can be given. The non-detection of this line in Longmore 4 and its weakness in K1-16 suggest an F abundance not higher than solar. The result for HS2324+397 is not very strict because of poor $\mathrm{S} / \mathrm{N}$ of the spectra and a strong contamination of the $\mathrm{FV}$ multiplet by interstellar $\mathrm{H}_{2}$ lines. Figure 2 displays the spectra of all five $\mathrm{H}$-rich central stars from our sample. The F VI $1139.5 \AA$ line profile for NGC 6853 is unique in being contaminated by warm $\mathrm{H}_{2}$ along the line of sight. The F VI line is bracketed by weak $\mathrm{H}_{2}$ features that account for at most half of the observed absorption on either side of the F VI line. 


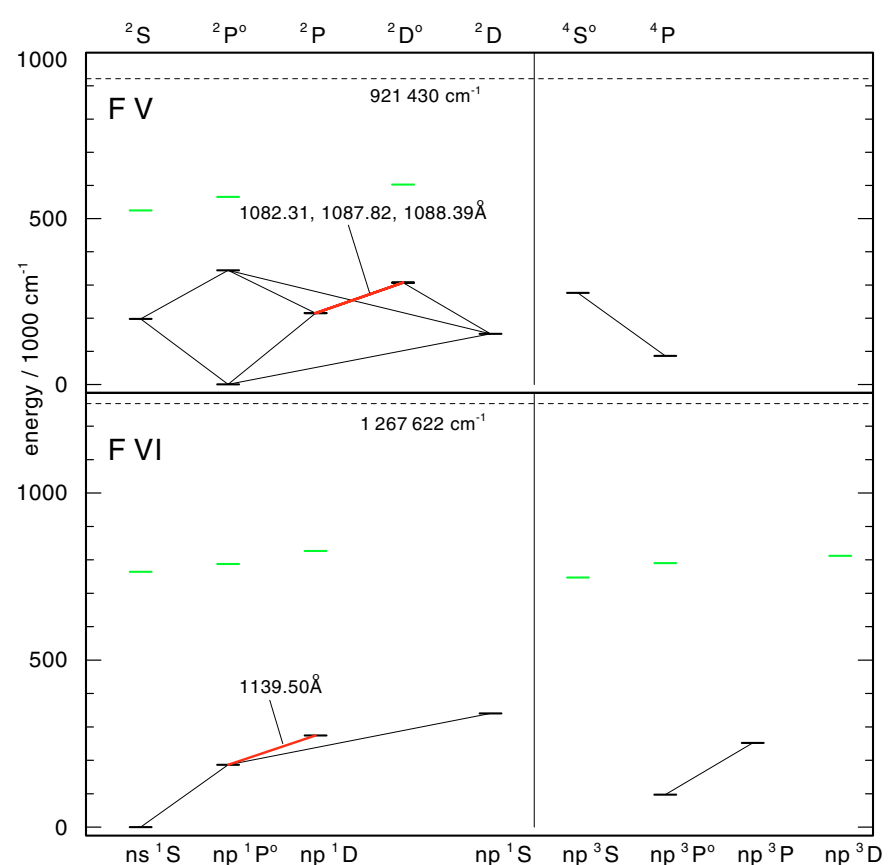

Fig. 3. Grotrian diagrams of our F V and F VI model ions. All levels linked by line transitions are computed in NLTE, others are LTE levels. Transitions observed in the FUSE spectra are marked. All other lines are located in the EUV, inaccessible with FUSE.

Figure 4 shows those four spectra in which we could identify the F V multiplet. They are from two H-rich central stars and from two PG1159 stars, all of which are relatively "cool" objects, for which the ionization balance shifts from F VI towards FV when compared to the hotter objects. All three lines of this multiplet are well matched by the model for PG1424+535 with the F abundance kept fixed from the fit to the F VI line, whereas the spectrum for PG1707+427 appears too noisy for a quantitative comparison. In contrast, the two computed F V lines at 1087.82 $\AA$ and 1088.39 $\AA$ for the two H-rich stars (NGC 1535 and NGC 1360) appear much too weak compared to the observation. But the third line component, at $1082.31 \AA$, is not detectable in these stars, which is in agreement with the computed weak profiles. According to the models, the components at 1082.31 $\AA$ and 1088.39 $\AA$ should be similar in strength as is the case with PG1424+535. The reason for the unexpectedly strong components at $1087.82 \AA$ 1088.39 $\AA$ in NGC 1535 and NGC 1360 remains unexplained.

The $\mathrm{F}$ abundance in the models was varied in steps of 0.5 dex. The derived $\mathrm{F}$ abundances are given in Tables 1 and 2 . We estimate the error of our abundance analysis to $0.5 \mathrm{dex}$, taking into account possible errors in the values for $T_{\text {eff }}$ and $\log g$, which are of the order $10 \%$ and 0.3 dex, respectively. For NGC 7094 and Abell 78 we must accept larger errors, maybe 1 dex, for the reasons mentioned above.

\section{Results and discussion}

Our fluorine abundance analysis can be summarized as follows (Fig. 5). We have identified fluorine lines in five H-rich central stars and in eight H-deficient PG1159 stars. Within error limits, the hydrogen-rich central stars have solar F abundances,

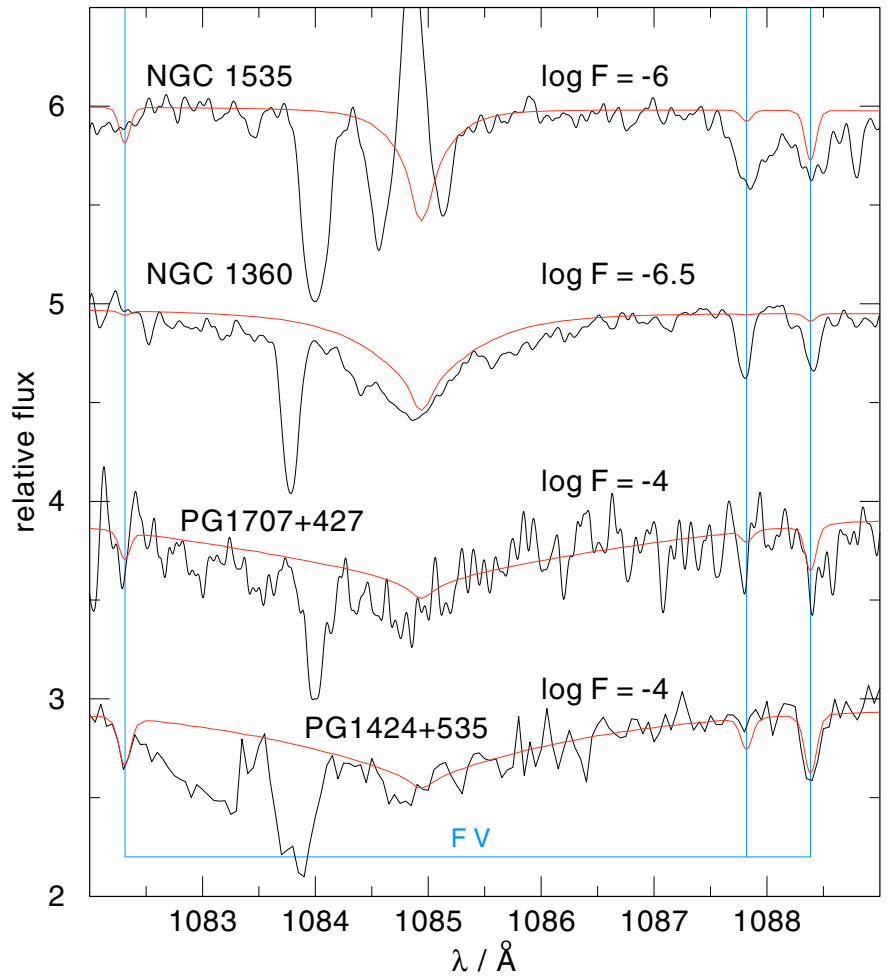

Fig. 4. Identification of a F V multiplet in two H-rich central stars (upper two spectra) and two PG1159 stars. The broad absorption line centered around $1085 \AA$ is from He II. The deep asymmetric depression over 1082-1083.5 $\AA$ in PG $1424+535$ is spurious.

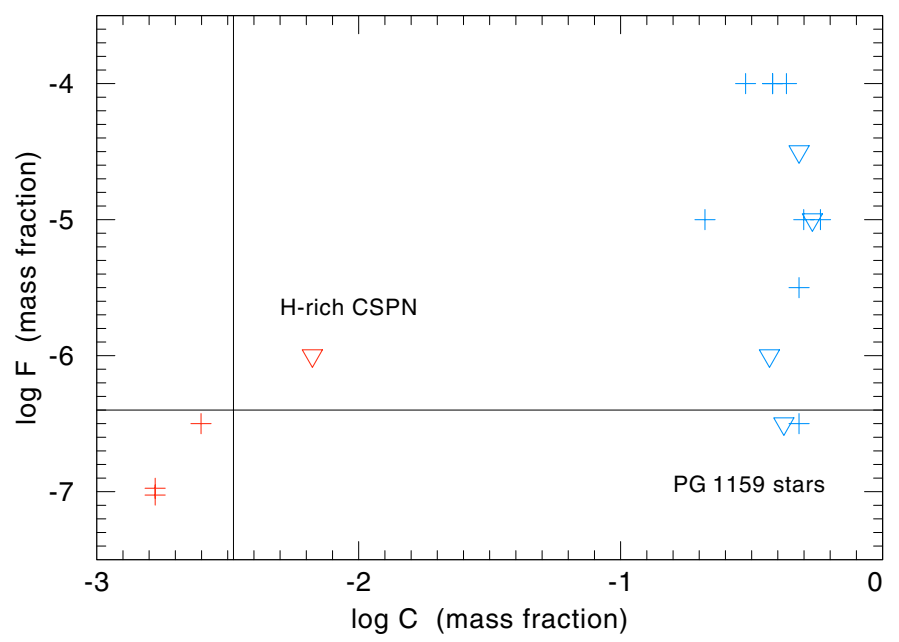

Fig. 5. The fluorine abundance as a function of the carbon abundance in our program stars (except for NGC 1535, for which no C abundance is available). The C-rich PG1159 stars are in general fluorine enriched in contrast to the (essentially) C-normal central stars. The vertical and horizontal lines denote the solar $\mathrm{C}$ and $\mathrm{F}$ abundance values, respectively. Typical errors for abundances are $0.3-0.5$ dex. Triangles denote stars for which only upper limits for F could be found.

i.e. $\log F=-6.4$. Most of the PG1159 stars show extreme F overabundances, ranging from 10-250 times solar ( $\log \mathrm{F}=$ $-5.4, \ldots,-4)$. There is one PG1159 star with a solar F abundance (K1-16) and another one with an upper limit of solar abundance (Longmore 4). By comparing the CSPN (which 
have essentially solar C abundances) and the C-rich PG1159 stars we find that the general trend of increasing $\mathrm{F}$ abundance with increasing $\mathrm{C}$ abundance is in accordance with nucleosynthesis modeling (Goriely \& Mowlavi 2000). But it must be stated that appropriate modeling for PG1159 stars, i.e. taking into account a late He-shell flash, is still lacking.

Concerning the H-rich stars we can conclude that the original $\mathrm{F}$ content in the stellar envelopes remains essentially unchanged. No enrichment due to dredge-up of F, which might have been synthesized in the AGB star, has occurred. Jorissen et al. (1992) found in their AGB star sample that the F enrichment is correlated with the enrichment of C. Matching to this, our five central stars are not significantly C-rich, see Table 2 where we list the $\mathrm{C} / \mathrm{O}$ ratio determined in a recent analysis of FUSE and HST spectra (Traulsen et al. 2005). The mass range $0.56-0.65 M_{\odot}$, covered by our CSPN, corresponds to an initialmass range of 1.2-2.6 $M_{\odot}$ (Weidemann 2000). According to the model calculations of Lugaro et al. (2004), a significant F enrichment on the AGB star surface can only be expected for stars with an inital mass larger than about $2.5 M_{\odot}$. This is in accordance with our abundance determinations. The iron abundance in the H-rich stars appears to be solar (Hoffmann et al. 2005), which is consistent with these findings.

A truly remarkable result is the high $\mathrm{F}$ overabundance found in the PG1159 stars. This strongly corroborates the idea, that these stars show intershell matter of the precursor AGB star on their surface. Herwig et al. (1999) were able to quantitatively explain the surface abundances of $\mathrm{He}, \mathrm{C}, \mathrm{O}$, and $\mathrm{Ne}$ as a consequence of a late He-shell flash. According to AGB stellar models, $\mathrm{F}$ is also strongly enriched in the intershell, so that the detected overabundances in the PG1159 stellar spectra fit qualitatively well into the overall picture. The detected deficiency of iron in PG1159 stars (Miksa et al. 2002) can also be understood. Neutron captures in the s-processing environments transform Fe into heavier elements and deplete the Fe content in the He-rich intershell. Hence, dredge-up of that intershell matter resulting from the late He-shell flash explains both the observed iron deficiency and the fluorine enrichment in the atmospheres of PG1159 stars.

What are the implications from the PG1159 star analyses for the modeling of nucleosynthesis in thermally pulsing AGB stars? The models of Lugaro et al. (2004) predict that the $\mathrm{F}$ enrichment in the intershell is a strong function of the initial stellar mass. For solar metallicity models, it is maximal at $M=3 M_{\odot}$, with $\log \mathrm{F}=-4.2$. It drops to $\log \mathrm{F}=-5$ for $M=5 M_{\odot}$, and to $\log \mathrm{F}=-6.2$ for a $M=6.5 M_{\odot}$ star. This variety of possible $\mathrm{F}$ intershell abundances is in agreement with our results for the PG1159 stars. Even K1-16 with a solar $\mathrm{F}$ abundance could be explained as the descendant of a particularly massive star, say $7-8 M_{\odot}$, however, the spectroscopically determined mass $\left(M=0.59 M_{\odot}\right)$ points to a less massive progenitor $\left(M=2 M_{\odot}\right.$, using the initial-final-mass relation of Weidemann 2000). Generally, we do not see a trend of the F abundance in PG1159 stars with the initial stellar mass (our sample covers the range $M_{i}=0.8-4.5 M_{\odot}$ ).

Hence, aside from this particular problem, the $\mathrm{F}$ intershell abundances in the Lugaro et al. (2004) models are large enough to explain even the highest overabundances observed in
PG1159 stars. In contrast, these authors conclude that their intershell abundances are not high enough to explain the F overabundances found in AGB stars. When mixed from the intershell into the H-rich convective surface layer, F gets dredged up to the surface, but is too diluted to match the large overabundances reported by Jorissen et al. (1992).

Although our results seem to confirm that the $\mathrm{F}$ enrichment in the intershell is described correctly by stellar models, this may be a chance coincidence. Without proper modeling including a detailed nuclear network it is not clear how the $\mathrm{F}$ abundance is changed during the event of a late helium-shell flash. Further work in this direction is urgently needed.

Acknowledgements. UV data analysis in Tübingen is supported by the DLR under grant 50 OR 0201. J.W.K. is supported by the FUSE project, funded by NASA contract NAS5-32985. We thank Falk Herwig (LANL) for helpful discussions on evolutionary aspects, and Alexander Kramida (NIST) for his advice on atomic data. We thank the referee, A. Jorissen, for constructive remarks.

\section{References}

Cunha, K., Smith, V. V., Lambert, D. L., \& Hinkle, K. H. 2003, AJ, 126, 1305

Dreizler, S., \& Heber, U. 1998, A\&A, 334, 618

Dreizler, S., Werner, K., \& Heber, U. 1997, in Planetary Nebulae, ed. H. J. Habing, \& H. J. G. L. M. Lamers, IAU Symp. (Kluwer), 180, 103

Goriely, S., \& Mowlavi, N. 2000, A\&A, 362, 599

Grevesse, N., \& Sauval, A. J. 1998, Space Sci. Rev., 85, 161

Herwig, F., Blöcker, T., Langer, N., \& Driebe, T. 1999, A\&A, 349, L5 Hoffmann, A. I. D., Traulsen, I., Dreizler, S., et al. 2005, in White Dwarfs, ed. D. Koester, S. Moehler, ASP Conf. Ser., in press

Jorissen, A., Smith, V. V., \& Lambert, D. L. 1992, A\&A, 261, 164

Koesterke, L., \& Werner, K. 1998, ApJ, 500, L55

Kruk, J. W., \& Werner, K. 1998, ApJ, 502, 858

Lugaro, M., Ugalde, C., \& Karakas, A. I., et al. 2004, ApJ, 615, L934

Méndez, R. H., Kudritzki, R. P., \& Herrero, A. 1992, A\&A, 260, 329

Meynet, G., \& Arnould, M. 2000, A\&A, 355, 176

Miksa, S., Deetjen, J. L., Dreizler, S., et al. 2002, A\&A, 389, 953

Mowlavi, N., Jorissen, A., \& Arnould, M. 1998, A\&A, 334, 153

Rauch, T., \& Werner, K. 1997, in The Third Conference on Faint Blue Stars, ed. A. G. D. Philip, J.Liebert, R. A. Saffer (Schenectady, NY: L. Davis Press), 217

Renda, A., Fenner, Y., Gibson, B. K., et al. 2004, MNRAS, 354, 575

Seaton, M. J., Yan, Y., Mihalas, D., \& Pradhan, A. K. 1994, MNRAS, 266, 805

Traulsen, I., Hoffmann, A. I. D., Dreizler, S., et al. 2005, in White Dwarfs, ed. D. Koester, \& S. Moehler, ASP Conf. Ser., in press

Weidemann, V. 2000, A\&A 363, 647

Werner, K. 2001, in Low Mass Wolf-Rayet Stars: Origin and Evolution, ed. T. Blöcker, L. B. F. M. Waters, \& A. A. Zijlstra, Ap\&SS, 275, 27

Werner, K., \& Heber, U. 1991, A\&A, 247, 476

Werner, K., Heber, U., \& Hunger, K. 1991, A\&A, 244, 437

Werner, K., Hamann, W.-R., Heber, U., et al. 1992, A\&A, 259, L69

Werner, K., Dreizler, S., Heber, U., et al. 1996, A\&A, 307, 860

Werner, K., Rauch, T., Reiff, E., Kruk, J. W., \& Napiwotzki, R. 2004a, A\&A, 427, 685

Werner, K., Rauch, T., Barstow, M. A., \& Kruk, J. W. 2004b, A\&A, 421, 1169

Woosley, S. E., \& Weaver, T. A. 1995, ApJS, 101, 181 